\title{
Use of Dried Blood Spots for Estimating Children's Exposures to Heavy Metals in Epidemiological Research
}

\author{
William E Funk ${ }^{1^{*}}$, Joachim D Pleil ${ }^{2}$, Dana J Sauter ${ }^{3}$, Thomas W McDade ${ }^{4}$ and Jane L Holl ${ }^{1}$ \\ ${ }^{1}$ Department of Preventive Medicine, Northwestern University, Chicago, IL, USA \\ ${ }^{2}$ United States Environmental Protection Agency, National Exposure Research Laboratory/ORD, Research Triangle Park, NC, USA \\ ${ }^{3}$ Cabot Microelectronics, Aurora, IL USA \\ ${ }^{4}$ Department of Anthropology and Institute for Policy Research, Northwestern University, Evanston, IL, USA
}

\begin{abstract}
Background: Children's exposures to arsenic (As), lead $(\mathrm{Pb})$, mercury $(\mathrm{Hg})$, and cadmium (Cd) are of particular concern in early-life. Exposures to heavy metals are traditionally measured in whole venous blood, which is costly and invasive. As an alternative we describe a method for quantifying $\mathrm{As}, \mathrm{Pb}, \mathrm{Hg}$, and $\mathrm{Cd}$ in dried blood spot (DBS) samples.

Objectives: To validate a method for quantifying levels of $\mathrm{As}, \mathrm{Pb}, \mathrm{Hg}$, and $\mathrm{Cd}$ in finger-stick DBS samples. Background metal contamination in blood collection cards poses a challenge for quantifying heavy metals in DBS samples. Here we report a method to remove background contamination from the filter paper prior to blood collection to improve assay precision.
\end{abstract}

Methods: Matched samples of venous blood and finger-stick DBS samples were collected from 82 children ages 1-21. Whole venous blood samples were also applied to pre-cleaned and untreated blood collection cards. All samples were analyzed for $\mathrm{As}, \mathrm{Pb}, \mathrm{Hg}$, and $\mathrm{Cd}$ using inductively coupled plasma mass spectrometry (ICP-MS).

Results: Matched venous blood and finger-stick DBS samples from untreated cards were significantly correlated, but with relatively weak $R^{2}$ values of $0.083,0.186,0.498$, and 0.022 for $\mathrm{As}, \mathrm{Cd}, \mathrm{Hg}$, and $\mathrm{Pb}$, respectively. When blood collection cards were decontaminated prior to blood collection the correlations between venous blood and DBS samples were highly significant, with $\mathrm{R}^{2}$ values of $0.66,0.99,0.98$, and 0.94 for $\mathrm{As}, \mathrm{Pb}, \mathrm{Hg}$, and $\mathrm{Cd}$, respectively.

Conclusions: Standard blood collection cards contain significant and highly variable background levels of heavy metals. Once blood collection cards are treated to remove residual contamination, DBS sampling can be used as a minimally-invasive alternative to venipuncture to estimate exposures to toxic metals.

Keywords: Dried blood spots; Heavy metals; Children

\section{Introduction}

The discipline of environmental exposure science is intricately linked with epidemiological investigations for protecting public health $[1,2]$. Historically, methods were focused on measurements of environmental media used to estimate potential human exposure through various uptake pathways. More recently, there has been a shift towards incorporating biomarker data as a more direct link to exogenous exposures through measurements from human biological media such as blood, breath, and urine for exploring the concept of the human exposome-representing all chemical exposures from conception throughout life [3-5]. The combined use of biomarkers of exposure and biomarkers of effect has also become a central theme for linking the external environment to potential adverse health outcomes [6-8]. There are now large databases such as the U.S. National Health and Nutrition Examination Survey (NHANES), the German Environmental Survey (Ger ES), The Korea National Health and Nutrition Examination Survey (KNHANES), and the Canadian Health Measures Study (CHMS) that are making such data mining readily available to the research community [9-12]. These are based on detailed analyses of thousands of "snapshot" measures from stratified random selections of subjects from the general populations of the respective countries. Supplementing such information with repeat measures and many more subjects will provide an ever-improving statistical understanding of environmental exposures, onboard dose, environmental metabolomics, and ultimately public health sustainability [13-16].

Currently, a major issue is the exposure of children to heavy metals, which are of particular concern early in development. In fact, $\mathrm{As}, \mathrm{Pb}, \mathrm{Hg}$, and $\mathrm{Cd}$ are listed as the $1^{\text {st }}, 2^{\text {nd }}, 3^{\text {rd }}$, and $7^{\text {th }}$ most important hazardous substances on the Agency for Toxic Substances and Disease Registry's 2013 CERCLA priority list of 275 substances, respectively. Exposure to heavy metals can occur through a variety of exposure routes, including inhalation as dust and fumes $[17,18]$, and ingestion from food and water [19-21], and can cause a wide spectrum of health problems including convulsions, coma, renal failure, injuries to the lungs and neurologic system, memory loss, delirium, diabetes, kidney damage, and a variety of cancers [22]. In addition, heavy metals can cross the placental barrier during pregnancy resulting in toxic exposures during highly susceptible periods of fetal development [23-25]. Yet, the health impacts of exposures to toxic metals during all stages of early development are not well understood due to the paucity of in vivo human data. Thus, additional studies are critically needed using emerging exposure assessment tools.

*Corresponding author: William E Funk, Department of Preventive Medicine, Feinberg School of Medicine, Northwestern University, $680 \mathrm{~N}$ Lake Shore Drive, Suite 1400, Chicago, IL 60611, USA, Tel: 312503 4092; E-mail: w-funk@northwestern.edu

Received July 02, 2015; Accepted July 16, 2015; Published July 24, 2015

Citation: Funk WE, Pleil JD, Sauter DJ, McDade TW, Holl JL (2015) Use of Dried Blood Spots for Estimating Children's Exposures to Heavy Metals in Epidemiological Research. J Environ Anal Toxicol S7: 002. doi:10.4172/2161-0525.S7-002

Copyright: ( 2015 Funk WE, et al. This is an open-access article distributed under the terms of the Creative Commons Attribution License, which permits unrestricted use, distribution, and reproduction in any medium, provided the original author and source are credited. 
Ideally, exposure to toxic metals should be monitored over time to evaluate changes that precede adverse health events and follow environmental exposures longitudinally. However, collecting blood samples with venipuncture in community-based research suffers from logistical obstacles (medical personnel, specialized containers, infectious wastes, refrigeration, etc.) and also from low acceptance in pediatric and younger populations [26]. As an alternative, dried blood spots (DBS)-drops of capillary whole blood collected from a finger or heel stick-can be used as a minimally-invasive and low cost alternative to invasive venipuncture [27].

The use of DBS sampling for screening newborns for metabolic disorders began in the 1960s [28], and archived residual newborn DBS have been used as an innovative resource for assessing certain environmental exposures [29,30]. In a previous study, we explored this resource for retrospective analysis of heavy metals $(\mathrm{As}, \mathrm{Cd}, \mathrm{Hg}$, and $\mathrm{Pb}$ ) but found that the standard blood collection cards (i.e., Whatman 903 Protein Saver cards) contain significant and variable background contamination for these elements [31]. To address this challenge, here we present two distinct approaches to 1) investigate the use of archived existing DBS samples for inferring systemic heavy metals exposure (retrospective studies), and 2) interpret the improvement in results that might be achieved with pre-cleaned blood collection cards designed specifically for collecting samples for metals analysis (prospective studies).

\section{Methods}

\section{Blood collection and human participants}

Eighty-two children from infancy to age 21 were recruited from the Anne and Robert H. Lurie Children's Hospital of Chicago (formerly Children's Memorial Hospital of Chicago) to participate in our study. Children were recruited and consented during regularly scheduled hospital visits, and volumes of blood that had already been drawn from each child were verified to ensure that no monthly draw limits were approached. Once consented, $5 \mathrm{~mL}$ of venous blood was collected by a phlebotomist in $7 \mathrm{~mL}$ metal-free vacutainers with EDTA as an anticoagulant and the tubes were gently inverted 8-10 times to ensure proper mixing. In addition, finger-stick DBS samples were collected at the time of phlebotomy by pricking the child's middle or ring finger using sterile single-use micro-lancets. Five drops of blood were collected on Whatman \#903 Protein Saver cards, and the specimens were allowed to dry uncovered at room temperature for a minimum of 4 hours. DBS samples were then placed in plastic bags with desiccants, and all blood samples were stored at $-20^{\circ} \mathrm{C}$ prior to shipment to Northwestern University. Once received in the laboratory all samples were frozen at $-80^{\circ} \mathrm{C}$ until assayed. This study was reviewed and approved by the Northwestern Institutional Review Board.

\section{Treatment of blood collection cards to remove metal contamination}

Five one-inch wide strips of Whatman \#903 Protein Saver cards were removed using ceramic scissors (part number, VWR, Atlanta, GA) that were washed in $5 \%$ acetic acid $(\mathrm{v} / \mathrm{v})$ solution prior to use. The excised strips of filter paper, each containing five circular blood collection guidelines, were placed in a $1 \mathrm{~L}$ Nalgene metal-free bottle (Fisher Scientific, Pittsburgh, PA) containing 5\% ultrapure grade nitric acid and 5\% ultrapure grade hydrochloric acid (Sigma Aldrich, St. Louis, MO) in $18.2 \mathrm{~m} \Omega$ deionized water. The filter paper strips were incubated for 90 minutes at room temperature on a shaker table at 200 $\mathrm{rpm}$. The solution was then decanted from the bottle, and the bottle was filled with $1 \mathrm{~L}$ of $18.2 \mathrm{~m} \Omega$ deionized water, and incubated for an additional 15 minutes, then followed by two additional water rinses. The cleaned filter paper strips were then removed using acid-washed Teflon tweezers and were dried overnight at room temperature.

\section{Spiked venous blood on DBS cards}

Forty previously-frozen whole venous blood samples were randomly selected from the initial 82 and were spotted onto precleaned and untreated strips of Whatman \#903 filter paper in $60 \mu \mathrm{L}$ aliquots. The strips were suspended horizontally above the laboratory bench top and were covered with $4 \mathrm{~L}$ beakers to protect the samples from contamination. The blood was dried for four hours at room temperature, and then transferred to trace-metal Nalgene plastic bags (Fisher Scientific, Pittsburgh, PA) and stored at $-80^{\circ} \mathrm{C}$ until assayed. We recognize the distribution of blood across the filter paper may have been altered due to cells being lysed during the freezing process. However, this did not impact our ability to estimate blood volumes since volumes were based on excising entire blood spots, as opposed to punches of blood that have assumed blood volumes.

\section{DBS sample processing}

DBS samples were extracted using the procedure described by Funk et al. [31], with minor modifications. In short, entire DBS specimens were excised with acid-washed ceramic scissors using the printed circles on the blood collection cards as a guide. The volume of blood in an intact DBS is approximately $60 \mu \mathrm{L}$. However, to account for blood volume variation between samples, the dried mass of each sample was normalized to the mean mass of all of the excised samples using previously described methods [31]. A similar approximate size of a blank filter paper was excised from each card near each blood sample to evaluate background metal contamination in the filter paper. The mass of each blank was also normalized to the mean mass of all of the excised blanks. DBS and filter paper blanks were weighed in $15 \mathrm{~mL}$ metal-free polypropylene centrifuge tubes (VWR, Atlanta, GA). An extraction solution was prepared using $5 \%$ ultrapure grade acetic acid and $0.01 \%$ ultrapure grade Triton X-100 (Fisher Scientific, Pittsburgh, PA) in $18.2 \mathrm{~m} \Omega$ deionized water. Two hundred ppb of Au was added to amalgamate $\mathrm{Hg}$ and prevent analyte loss throughout the procedure (Inorganic Ventures, Christiansburg, VA), and five ppb of indium, bismuth, and yittrium were added to the extraction solution as internal standards (Inorganic Ventures, Christiansburg, VA). Approximately $1.5 \mathrm{~mL}$ of extraction solution was then added directly to each vial and the accurate volumes of the extraction solutions were determined by mass. DBS samples and filter paper blanks were then centrifuged at 3600 $\mathrm{xg}$ for 2 minutes and incubated for 90 minutes at room temperature on a shaker table at $300 \mathrm{rpm}$. Prior to analysis the centrifuge tubes were inverted and manipulated to adhere the filter paper to the side of the tubes in order to remove them from the blood extracts.

\section{Venous blood processing}

Using acid washed pipette tips, $50 \mu \mathrm{L}$ of venous whole blood was spiked directly into $15 \mathrm{~mL}$ metal-free polypropylene centrifuge tubes containing $1.5 \mathrm{~mL}$ of extraction solution (described above). The blood extracts were then centrifuged at $3600 \times \mathrm{g}$ for 2 minutes and incubated for 90 minutes at room temperature on a shaker table at $300 \mathrm{rpm}$.

\section{Phase 1: DBS vs. whole venous blood (retrospective applications)}

Eighty-two matched finger stick DBS (i.e., standard DBS cards) and whole venous blood samples were evaluated for potential use for 
Citation: Funk WE, Pleil JD, Sauter DJ, McDade TW, Holl JL (2015) Use of Dried Blood Spots for Estimating Children's Exposures to Heavy Metals in Epidemiological Research. J Environ Anal Toxicol S7: 002. doi:10.4172/2161-0525.S7-002

retrospective analysis, that is, using cards previously collected without special procedures for removing background metals. The samples were individually matched as measurements of blank filter paper, DBS, and venous whole blood; they were processed to determine the geometric mean (GS) and geometric standard deviation (GSD) for each metal to characterize their lognormal distributions. The DBS data were individually corrected (subtracted) with their respective blank values for each metal. Standard percentile values were directly observed from tabulated data, and subsequently, scatterplots and regressions were evaluated for association between the "gold standard" venous blood and DBS samples. Correlations between venous blood and DBS samples were evaluated with and without blank background subtraction.

\section{Phase 2: DBS sampling using pre-cleaned vs. untreated blood collection cards (prospective applications)}

Whole venous blood spotted onto 40 matched pre-cleaned and untreated Whatman\#903 filter strips were extracted and analyzed to assess the potential for using DBS sampling in prospective studies specifically designed for heavy metal analysis. Here, one set of cards was preprocessed to remove pre-existing metals contamination to provide a uniformly clean substrate. Scatterplots and regressions were evaluated for association between the pre-cleaned and untreated DBS samples.

\section{Mass spectrometry}

Concentrations of $\mathrm{As}, \mathrm{Pb}, \mathrm{Hg}$, and $\mathrm{Cd}$ were quantified using a Thermo Fisher $\mathrm{X}$ Series II Inductively Couple Plasma Mass Spectrometer (ICP-MS). Metal concentrations were determined using a five-point calibration curve for each analyte. For $\mathrm{Pb}$, three isotopes were measured and summed ( $\mathrm{m} / \mathrm{z}: 206,207$, and 208). Arsenic, Hg, and Cd, were quantified using single isotopes with $\mathrm{m} / z$ of 75,202 , and 111 , respectively. In addition to the samples and paired card blanks, quality control samples were run along with each batch, consisting of a matrix blank, a trace element whole blood reference (Clin Chek, Munich, Germany), and a trace element whole blood reference spiked onto Whatman \#903 filter paper.

\section{Statistics and graphing}

Measurement data were organized using MS Excel spreadsheet software (Microsoft Corp., Redmond, WA); statistical analyses and graphing were performed using both Excel and Graph Pad Prism (Graph Pad Software, Inc., La Jolla, CA). All data sets were individually examined for summary statistics (mean, standard deviation, median and coefficient of variation) and characterized as exhibiting lognormal distribution (32). The few below detection limit (BDL) values were imputed using the limit of detection (LoD) value; this was necessary for 3 of 1134 total measurements.

\section{Results and Discussion}

\section{DBS vs. whole venous blood (retrospective applications)}

Summary statistics for heavy metal concentrations (in $\mathrm{ppb}$ ) from blank cards, venous whole blood samples, DBS and background corrected DBS values are provided in Table 1. The geometric mean (GM) and geometric standard deviation (GSD) are calculated under the assumption of lognormal distribution [32]; the remaining statistics are strictly observational; that is, no particular underlying distributions are assumed. Entries of " $<\mathrm{LoD}$ " indicate that the value is below the level of detection, entries with "-_" occurring for the blank corrected (subtracted) DBS were negative. Due to individual zero or negative values, certain GM entries (As blanks, Cd blood, and all DBS corr.) were estimated by the observed median, rather than from direct calculation of log-transformed data (Table 1).

These summary data indicate that the whole blood and DBS method yield similar trends, but with noticeable mean level offsets presumably stemming from background metal concentrations inherent to the filter paper matrix. While use of pair-wise blank subtraction did not significantly improve results (described below), taken as groups, it is probably reasonable to apply a group "blank" correction.

\section{Individual samples evaluation}

We found that blank correction at the individual sample level is not particularly helpful. This observation is attributed to large spot-to-spot

\begin{tabular}{|c|c|c|c|c|c|c|c|c|c|c|}
\hline Metal Species & Sample & GM & GSD & Min & $5^{\text {th }} \%$ & $25^{\text {th }} \%$ & $50^{\text {th }} \%$ & $75^{\text {th }} \%$ & $95^{\text {th }} \%$ & Max \\
\hline \multirow{4}{*}{ As (ppb) } & Blanks & 0.05 & 6.68 & $<$ LoD & $<$ LoD & $<$ LoD & 0.05 & 0.66 & 1.08 & 2.61 \\
\hline & Blood & 8.83 & 1.18 & 6.03 & 6.81 & 7.96 & 8.94 & 9.70 & 11.12 & 16.28 \\
\hline & DBS & 9.40 & 2.21 & $<$ LoD & 5.75 & 6.89 & 8.25 & 11.24 & 30.39 & 50.95 \\
\hline & DBS Corr. & 7.97 & 2.26 & 0.00 & 5.18 & 6.58 & 7.97 & 11.02 & 30.36 & 50.95 \\
\hline \multirow{4}{*}{$\mathrm{Cd}(\mathrm{ppb})$} & Blanks & 0.14 & 1.43 & $<$ LoD & 0.05 & 0.12 & 0.13 & 0.16 & 0.24 & 0.65 \\
\hline & Blood & 0.10 & 1.85 & $<$ LoD & 0.00 & 0.05 & 0.10 & 0.15 & 0.28 & 0.48 \\
\hline & DBS & 0.32 & 2.73 & $<$ LoD & 0.12 & 0.18 & 0.29 & 0.48 & 1.52 & 2.71 \\
\hline & DBS Corr. & 0.14 & 3.98 & -- & -- & 0.05 & 0.14 & 0.32 & 1.37 & 2.71 \\
\hline \multirow{4}{*}{$\mathrm{Hg}(\mathrm{ppb})$} & Blanks & 0.10 & 2.43 & 0.04 & 0.04 & 0.05 & 0.09 & 0.16 & 0.37 & 2.86 \\
\hline & Blood & 0.45 & 1.97 & 0.13 & 0.15 & 0.28 & 0.45 & 0.69 & 1.40 & 2.10 \\
\hline & DBS & 0.53 & 1.68 & $<$ LoD & 0.25 & 0.35 & 0.51 & 0.72 & 1.20 & 8.04 \\
\hline & DBS Corr. & 0.36 & 1.99 & -- & 0.04 & 0.26 & 0.36 & 0.59 & 1.11 & 5.17 \\
\hline \multirow{4}{*}{$\mathrm{Pb}(\mathrm{ppb})$} & Blanks & 1.89 & 3.18 & 0.02 & 0.75 & 1.08 & 1.58 & 3.14 & 10.55 & 41.88 \\
\hline & Blood & 6.32 & 1.81 & 2.38 & 2.91 & 4.03 & 5.70 & 8.56 & 20.17 & 30.45 \\
\hline & DBS & 11.01 & 2.19 & $<$ LoD & 4.39 & 5.84 & 9.44 & 19.15 & 34.14 & 122.76 \\
\hline & DBS Corr. & 6.60 & 2.67 & -- & -- & 3.19 & 6.60 & 16.62 & 33.14 & 122.00 \\
\hline
\end{tabular}

Table 1: Summary statistics: Group-wise comparison of metal species and sample format; all DBS measurements are from standard Whatman \#903 cards ( $\mathrm{n}=82 / \mathrm{group}$ ). 
heterogeneity in background contamination within cards. In fact, there seems to be additional biasing effects from the cards occurring beyond random contamination, for which we have no explanation. Figures 1-4 show the direct comparisons between venous blood levels ( $\mathrm{x}$-axis) vs. finger stick DBS samples (y-axis), with and without individual background correction.

Visual inspection of the scatterplots shows that the relationship between the accepted blood measurement and the corresponding measurement in DBS samples is not much improved by within-card background correction. However, slopes are positive with statistical significance for all four metals indicating that there are indeed positive correlations between venous blood and DBS measurements. Table 2 shows these results as calculated in log space. Slopes are all less than 1.0; we attribute this to positive bias at lower absolute blood concentrations. The p-values for the slopes as different from zero are highly significant; however, as is obvious from the scatterplots, the $\mathrm{r}^{2}$ values demonstrate that there is appreciable scatter in the data.

These results demonstrate that there is little value in correcting individually for metals background as these levels are highly variable within a card. The best we could accomplish would be a form of blanket statistical subtraction for all cards that would show only statistically valid exceedances beyond the highest overall background levels. Such retrospective approaches will be explored in future work.

DBS sampling using pre-cleaned vs. untreated blood collection cards (prospective applications)

The focus of this part of the investigation was to determine if the background confounding could be resolved by pre-cleaning filter paper before use. Certainly, this is only possible for prospective type studies, where the cards can be treated before the blood is collected. We used 40 venous blood samples from individuals to test the precleaning method; again, the results from venous blood analyses were considered the accepted "gold standard" values, and were compared to the standard DBS samples and the DBS samples that were spotted onto pre-cleaned cards. Table 3 shows the summary evaluation of these results (analogous to the Table 1 results). Here we see that the summary and percentile statistics are much more aligned between the cleaned DBS substrate and the venous blood values (Table 3).

These summary data indicate an across the board improvement in linking DBS and venous blood metals content when using pre-cleaned cards, especially for $\mathrm{Hg}$ and $\mathrm{Pb}$. Taken as a group, there is no obvious (qualitative) bias.

\section{Individual samples evaluation}

As in the retrospective analysis section, it is important to assess the comparisons of the prospective data at the individual samples level. Again, we show the scatterplots, this time between the venous blood analyses (x-axis) and the measurements of the DBS samples from the pre-cleaned cards (y-axis).

These scatterplots using pre-cleaned cards demonstrate a marked improvement over their counterparts in the previous section using standard DBS collected on untreated cards. We note that there is a slight bias in the slopes below 1.0, which could be perceived as a small sink for trace metals created by the acid wash process. This is pure speculation; it is also possible that this is an artifact of linear regression of data that should have been log-transformed or a few influential outliers. Regardless, the cleaned cards provide a significant improvement and could be used effectively as a surrogate for venous whole blood analysis.

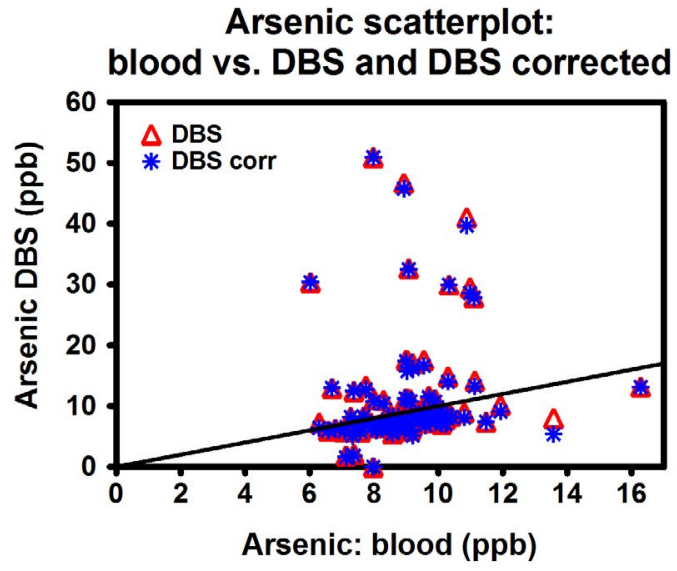

Figure 1: Scatterplots of arsenic: venous blood vs. DBS (triangle) and venous blood vs. DBS corrected for within card blank value (star). Straight line indicates perfect agreement.

Cadmium scatterplot: blood vs. DBS and DBS corrected

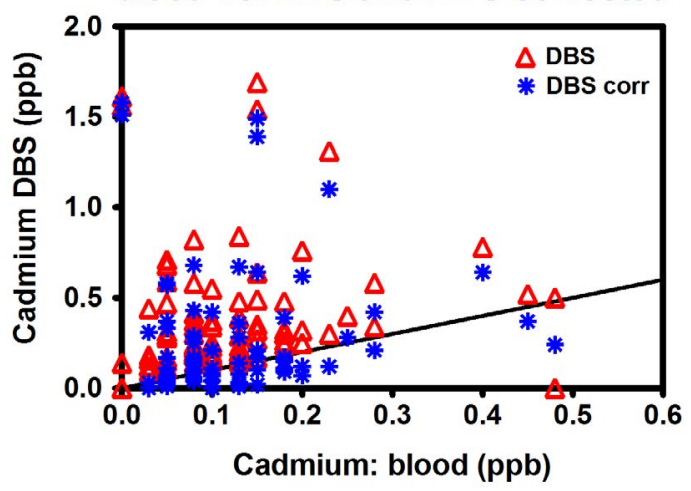

Figure 2: Scatterplots of cadmium: venous blood vs. DBS (triangle) and venous blood vs. DBS corrected for within card blank value (star). Straight line indicates perfect agreement.

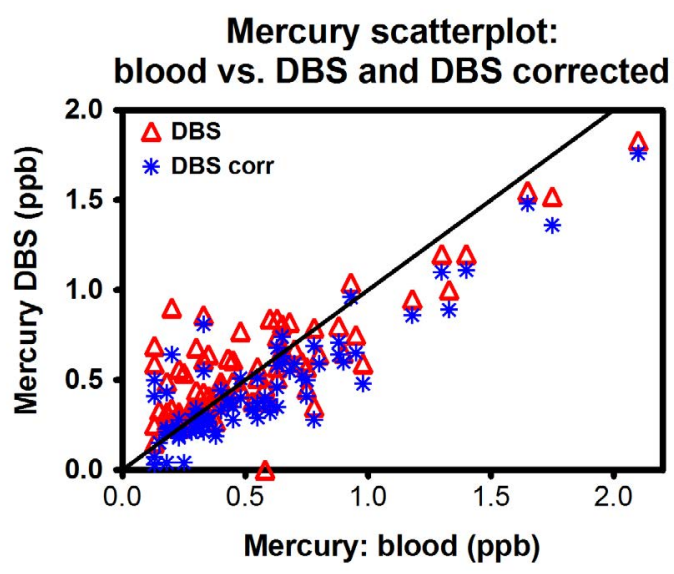

Figure 3: Scatterplots of mercury: venous blood vs. DBS (triangle) and venous blood vs. DBS corrected for within card blank value (star). Straight line indicates perfect agreement. 
Citation: Funk WE, Pleil JD, Sauter DJ, McDade TW, Holl JL (2015) Use of Dried Blood Spots for Estimating Children's Exposures to Heavy Metals in Epidemiological Research. J Environ Anal Toxicol S7: 002. doi:10.4172/2161-0525.S7-002

Page 5 of 9

\begin{tabular}{|c|c|c|c|c|}
\hline \multirow{2}{*}{ Metal Species } & Compare & Slope & p-value & sig. pos. \\
\hline \multirow{2}{*}{ As } & Blood vs. DBS & 0.983 & 0.009 & 0.083 \\
\hline & Blood vs. DBS corr & 0.89 & 0.022 & 0.065 \\
\hline \multirow{2}{*}{$\mathrm{Cd}$} & Blood vs. DBS & 0.392 & $<0.001$ & 0.186 \\
\hline & Blood vs. DBS corr & 0.698 & 0.002 & 0.134 \\
\hline $\mathrm{Hg}$ & Blood vs. DBS & 0.602 & $<0.001$ & yes \\
\hline & Blood vs. DBS corr & 0.872 & $<0.001$ & 0.575 \\
\hline $\mathrm{Pb}$ & Blood vs. DBS & 0.594 & $<0.001$ & 0.022 \\
\hline
\end{tabular}

Table 2: Summary statistics for scatterplot slopes of association between venous blood measurements and DBS measurements from standard Whatman \#903 cards ( $\mathrm{n}=82$ group).

\begin{tabular}{|c|c|c|c|c|c|c|c|c|c|c|}
\hline $\begin{array}{c}\text { Metal } \\
\text { Species }\end{array}$ & Sample & GM & GSD & Min & $5^{\text {tho }} \%$ & $25^{\text {th }} \%$ & $50^{\text {th }} \%$ & $75^{\text {th }} \%$ & $95^{\text {th }} \%$ & Max \\
\hline \multirow{3}{*}{ As (ppb) } & DBS orig & 5.68 & 2.68 & $<$ LoQ & 20 & 2.77 & 7.16 & 10.94 & 16.73 & 19.02 \\
\hline & DBS clean & 7.48 & 154 & 0.9 & 5.01 & 6.73 & 7.76 & 8.96 & 11.11 & 17.83 \\
\hline & Blood & 9.1 & 139 & 3.6 & 594 & 7.52 & 9.73 & 11.1 & 12.79 & 24 \\
\hline \multirow{3}{*}{$\mathrm{Cd}(\mathrm{ppb})$} & DBS orig & 0.09 & 1.8 & 0.12 & 0.15 & 0.18 & 0.23 & 0.27 & 0.45 & 0.9 \\
\hline & DBS clean & 0.09 & 1.77 & $<$ LoQ & 0.03 & 0.05 & 0.08 & 0.11 & 0.113 & 0.43 \\
\hline & Blood & 0.09 & 1.8 & 0.03 & 0.05 & 0.08 & 0.08 & 0.13 & 0.2 & 0.5 \\
\hline \multirow{3}{*}{$\mathrm{Hg}(\mathrm{ppb})$} & DBS orig & 2.41 & 1.44 & 1.68 & 1.68 & 1.92 & 2.22 & 2.74 & 3.85 & 11.82 \\
\hline & DBS clean & 0.59 & 1.76 & 0.18 & 25 & 0.4 & 0.63 & 0.81 & 1.49 & 1.9 \\
\hline & Blood & 0.43 & 224 & 0.08 & 0.13 & 0.23 & 0.44 & 0.73 & 1.65 & 1.98 \\
\hline \multirow{3}{*}{$\mathrm{Pb}(\mathrm{ppb})$} & DBS orig & 8.2 & 1.6 & 3.42 & 428 & 6.32 & 759 & 11.42 & 16.83 & 30.54 \\
\hline & DBS clean & 5.22 & 1.71 & 2.3 & 255 & 3.49 & 496 & 6.43 & 13.32 & 26.48 \\
\hline & Blood & 5.98 & 1.66 & 2.6 & 3.02 & 4.03 & 5.63 & 7.69 & 13.66 & 30.18 \\
\hline
\end{tabular}

Table 3: Summary statistics: Group-wise comparison of metal species and sample format; DBS measurements are from standard DBS samples, and from DBS samples collected on pre-cleaned cards; "Blood" refers to venous blood measurements ( $n=40 /$ group).

In the Table 3 below, we present the slope factor results comparing the linear regressions as drawn in Figures 5-8, and the presumed more robust slopes calculated in log-log space. The results are equivocal, with some slight improvement in slope comparisons for As, Cd, and $\mathrm{Pb}$ (Table 4).

\section{Predicting blood concentrations from DBS}

If we concede that the log-transformed regressions are slightly more robust in dealing with influential outliers, then we can estimate the concentration in the blood $\left(\mathrm{C}_{\text {blood }}\right)$ in terms of the measured value from the pre-cleaned DBS samples and the regression coefficients as:

$$
C_{\text {blood }}(D B S)=\exp \left\{\left[\ln (D B S)-b^{\prime}\right] / m^{\prime}\right\}
$$

where $\mathrm{m}^{\prime}=$ slope and $\mathrm{b}^{\prime}$ = intercept are the regression coefficients of the log-transformed data, analogous to the regressions performed using the raw data in Figures 5-8. One could also use the linear regressions from those figures in a standard calibration application in this format:

$$
C_{\text {blood }}(D B S)=(D B S-b) / m
$$

where $\mathrm{m}$ and $\mathrm{b}$ are the slope and intercept coefficients from the natural space regressions. The choice of regression space is not obvious.
If there are large influential outliers, then log-space is preferable, if however, there are many very low-level imputed values, they skew the low end unnecessarily and linear regression yields a more practical result.

The next step is to estimate the variability between the standard method (venous blood analysis) and the pre-cleaned DBS sampling method. Although we might designate the venous blood method to be the default, it too is subject to some level of variability, and so we need to make a decision as to how to interpret the comparisons. There are two paths: pronounce that the variability in the venous blood measurements is negligible (gold standard), or assume that we do not know and treat each method equally with respect to variance. A second decision is how to couch the results; we can either state error in terms of percent with respect to the concentration, or make a blanket statement of confidence limit across all levels. Either is acceptable, often the percent method is preferable as it is easier to compare disparate data, in our case, across different metal species. Table 5 shows the results from this regression exercise for both approaches.

The table demonstrates that the choice of prediction space is equivocal. The one anomaly is the slope/intercept for the prediction 


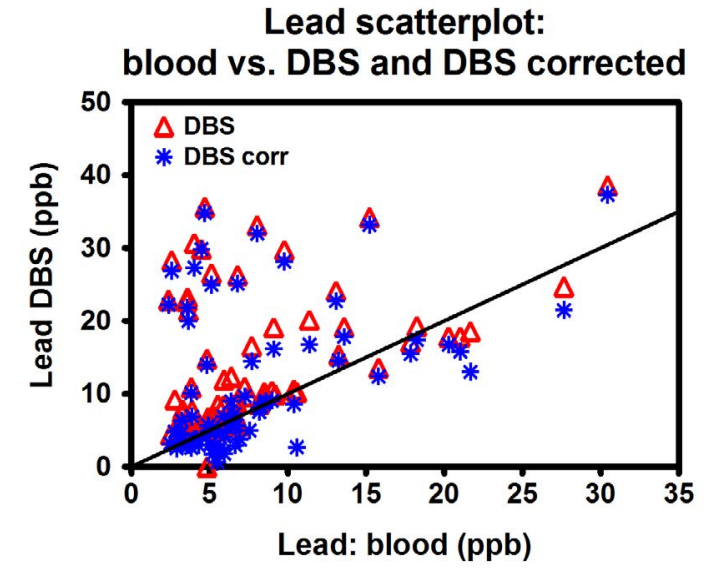

Figure 4: Scatterplots of lead: venous blood vs. DBS (triangle) and venous blood vs. DBS corrected for within card blank value (star). Straight line indicates perfect agreement.

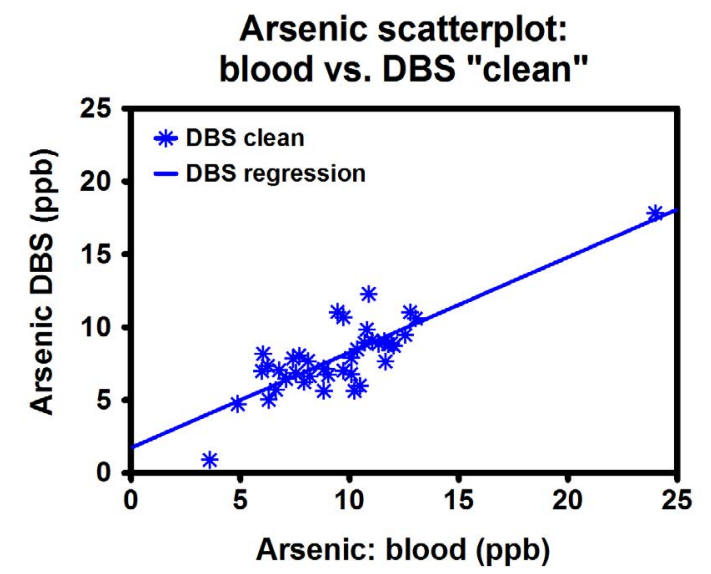

Figure 5: Scatterplot of lead data: venous blood vs. DBS on pre-cleaned card. Straight line indicates linear regression curve: $y(x)=0.6538(x)+1.733$ $\left(r^{2}=0.58\right)$.

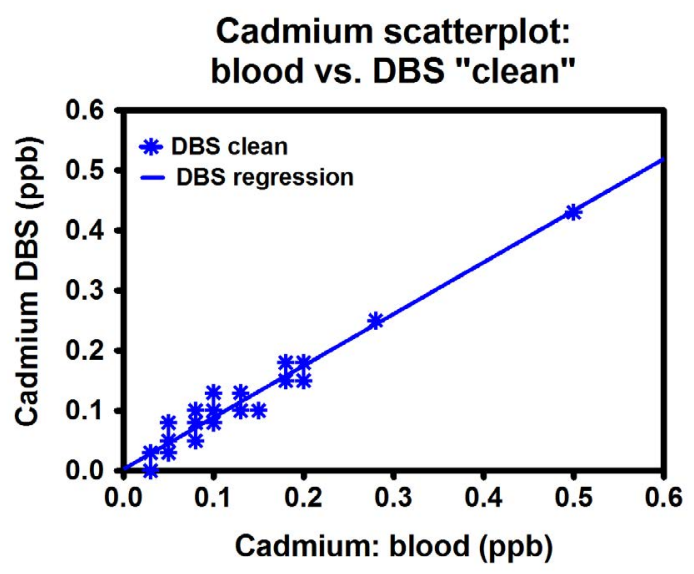

Figure 6: Scatterplot of cadmium data: venous blood vs. DBS on pre-cleaned card. Straight line indicates linear regression curve: $y(x)=0.8606(x)+0.0027$ $\left(r^{2}=0.86\right)$.

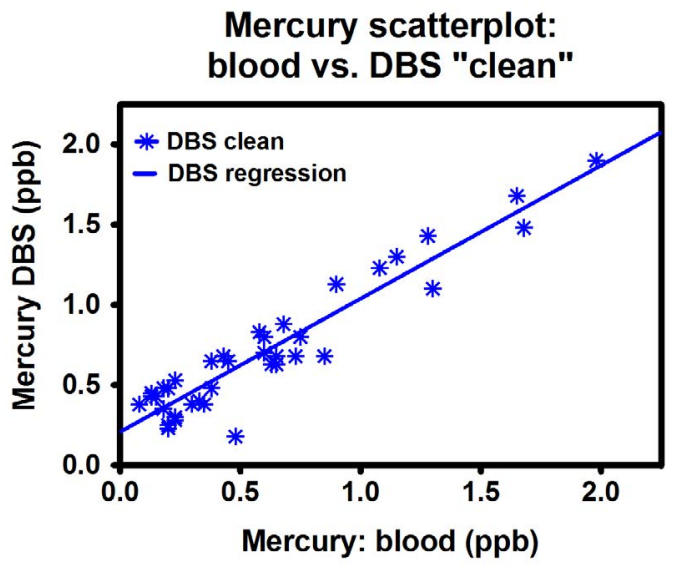

Figure 7: Scatterplot of mercury data: venous blood vs. DBS on pre-cleaned card. Straight line indicates linear regression curve: $y(x)=0.8308(x)+0.2081$ $\left(r^{2}=0.66\right)$.

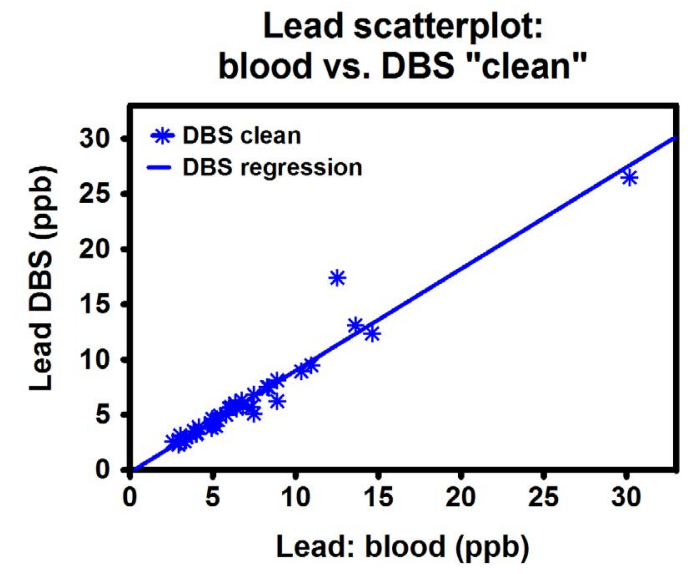

Figure 8: Scatterplot of lead data: venous blood vs. DBS on pre-cleaned card. Straight line indicates linear regression curve: $y(x)=0.9209(x)-0.2085$ $\left(r^{2}=0.96\right)$.

of As, where there is some clustering (see the scatterplot in Figure 5) that appears to shift the slope a bit. Overall, the scatter between the two methods has a prediction band error ranging from $\pm 21 \%$ to $\pm 76 \%$. This is not as dire as it initially appears. Consider for example that a DBS measurement of as at the median of $7.16 \mathrm{ppm}$ would indicate a blood level between 4.3 and 10.0 ppm with $95 \%$ confidence. Also, we need to consider that the venous blood levels are subject to error. As such, the relationship between cleaned DBS samples and venous blood measurements is useful for using one or the other.

\section{Conclusions}

Dried blood spots are proposed as a non-invasive and even self-administered alternative to sampling whole venous blood. The retrospective data from standard DBS cards are not suitable for metals analysis as they exhibit too much heterogeneity of background. However, all standard DBS measurements demonstrate a positive slope with respect to matched venous blood samples suggesting that there is at least some statistical, albeit noisy, relationship. It is possible to investigate this behavior in more detail with further measurements of 
Citation: Funk WE, Pleil JD, Sauter DJ, McDade TW, Holl JL (2015) Use of Dried Blood Spots for Estimating Children's Exposures to Heavy Metals in Epidemiological Research. J Environ Anal Toxicol S7: 002. doi:10.4172/2161-0525.S7-002

Page 7 of 9

\begin{tabular}{|c|c|c|c|c|c|}
\hline Metal Species & Compare & Slope & p-value & $\mathbf{r}^{2}$ & sig. pos. \\
\hline \multirow{2}{*}{ As } & Blood vs. DBS (lin.) & 0.654 & $<0.0001$ & 0.682 & yes \\
\hline & Blood vs. DBS (log) & 1.003 & $<0.0001$ & 0.58 & yes \\
\hline \multirow{2}{*}{$\mathrm{Cd}$} & Blood vs. DBS (lin.) & 0.861 & $<0.0001$ & 0.861 & yes \\
\hline & Blood vs. DBS (log) & 0.916 & $<0.0001$ & 0.858 & yes \\
\hline \multirow{2}{*}{$\mathrm{Hg}$} & Blood vs. DBS (lin.) & 0.831 & $<0.0001$ & 0.892 & yes \\
\hline & Blood vs. DBS (log) & 0.568 & $<0.0001$ & 0.658 & yes \\
\hline \multirow{2}{*}{$\mathrm{Pb}$} & Blood vs. DBS (lin.) & 0.844 & $<0.0001$ & 0.94 & yes \\
\hline & Blood vs. DBS (log) & 1.033 & $<0.0001$ & 0.956 & yes \\
\hline
\end{tabular}

Table 4: Summary statistics for scatterplot slopes of association between venous blood measurements and DBS measurements from DBS collected on pre-cleaned cards ( $n=40 /$ group), in both linear and log-transformed data regressions.

\begin{tabular}{|c|c|c|c|c|c|}
\hline Metal Species & Prediction Model & slope (m) & intercept (b) & $\mathbf{r}^{2}$ & $\%$ error $(+/-)$ \\
\hline \multirow{2}{*}{ As } & Blood vs. DBS (lin.) & 1.000 & -0.001 & 0.6819 & 47.6 \\
\hline & Blood vs. DBS (log) & 0.793 & 2.131 & 0.6186 & 38.8 \\
\hline \multirow{2}{*}{$\mathrm{Cd}$} & Blood vs. DBS (lin.) & 0.997 & -0.003 & 0.9498 & 50.5 \\
\hline & Blood vs. DBS (log) & 1.045 & -0.005 & 0.9535 & 40.2 \\
\hline \multirow{2}{*}{$\mathrm{Hg}^{*}$} & Blood vs. DBS (lin.) & 1.000 & -0.004 & 0.8921 & 76.5 \\
\hline & Blood vs. DBS (log) & 1.083 & -0.040 & 0.9113 & 62.7 \\
\hline \multirow{2}{*}{$\mathrm{Pb}$} & Blood vs. DBS (lin.) & 1.000 & -0.001 & 0.9399 & 21.5 \\
\hline & Blood vs. DBS (log) & 0.997 & 0.076 & 0.9393 & 21.2 \\
\hline
\end{tabular}

*5 low-level imputed values removed

Table 5: Prediction band results from calibrated "clean" DBS vs. venous blood regressions in linear and log-space.

the spatial heterogeneity within cards, and the population heterogeneity between cards.

Using pre-cleaned cards for prospective (non-invasive) sampling has promise. Based on these limited samples, we find excellent linearity for groups of samples, and that a single DBS sample can provide a bounded and useful estimate for the "true" blood level as illustrated in Table 5.

\section{Acknowledgments}

We thank K. Angevine for recruiting and consenting all of the participants that provided blood samples, and we are grateful to the children and their families who graciously supported this work. This research has been subjected to U.S. Environmental Protection Agency review and approved for publication. This study was supported through a Formative Research Grant from the National Children's Study (LOI3-BIO-01-A, Analysis of environmental chemicals in dried blood spots).

\section{Competing Financial Interests}

W Funk and T McDade filed a U.S. patent application, number 61/803674 "Detection of Heavy Metals in Dried Blood", on March 20, 2013.

\section{References}

1. Harper M, Weis C, Pleil JD, Blount BC, Miller A, et al. (2015) Commentary on the contributions and future role of occupational exposure science in a vision and strategy for the discipline of exposure science. Journal of exposure science and environmental epidemiology 25: 381-387.

2. Pleil JD, Blount BC, Waidyanatha S, Harper M (2012) Establishing exposure science as a distinct scientific discipline. J Expo Sci Environ Epidemiol 22: 317319

3. Bean HD, Pleil JD, Hill JE (2015) Editorial: new analytical and statistical approaches for interpreting the relationships among environmental stressors and biomarkers. Biomarkers 20: 1-4.

4. Rappaport SM, Smith MT (2010) Epidemiology. Environment and disease risks. Science 330: 460-461.
5. Wild CP (2005) Complementing the genome with an "exposome": the outstanding challenge of environmental exposure measurement in molecular epidemiology. Cancer epidemiology, biomarkers and prevention 14: 18471850.

6. Pleil JD, Sheldon LS (2011) Adapting concepts from systems biology to develop systems exposure event networks for exposure science research. Biomarkers 16: 99-105.

7. Sobus JR, Tan YM, Pleil JD, Sheldon LS (2011) A biomonitoring framework to support exposure and risk assessments. Sci Total Environ 409: 4875-4884.

8. Tan YM, Sobus J, Chang D, Tornero-Velez R, Goldsmith M, et al. (2012) Reconstructing human exposures using biomarkers and other "clues". J Toxicol Environ Health B Crit Rev 15: 22-38.

9. Park HA (2013) The Korea national health and nutrition examination survey as a primary data source. Korean J Fam Med 34: 79.

10. Sobus JR, DeWoskin RS, Tan YM, Pleil JD, Phillips MB, et al. (2015) Uses of NHANES biomarker data for chemical risk assessment: Trends, challenges, and opportunities. Environmental Health Perspectives.

11. Canadian Health Measures Survey 2015.

12. German Environmental Survey 2015.

13. Demetriou CA, Vineis $P(2015)$ Carcinogenicity of ambient air pollution: use of biomarkers, lessons learnt and future directions. J Thorac Dis 7: 67-95.

14. Miller MG (2007) Environmental metabolomics: A SWOT analysis (strengths weaknesses, opportunities, and threats). J Proteome Res 6: 540-545.

15. Pleil JD (2012) Categorizing biomarkers of the human exposome and developing metrics for assessing environmental sustainability. J Toxicol Environ Health B Crit Rev 15: 264-280.

16. Sørensen M, Autrup H, Møller P, Hertel O, Jensen SS, et al. (2003) Linking exposure to environmental pollutants with biological effects. Mutat Res 544: 255-271. 
Citation: Funk WE, Pleil JD, Sauter DJ, McDade TW, Holl JL (2015) Use of Dried Blood Spots for Estimating Children's Exposures to Heavy Metals in Epidemiological Research. J Environ Anal Toxicol S7: 002. doi:10.4172/2161-0525.S7-002

17. Barbee JY Jr, Prince TS (1999) Acute respiratory distress syndrome in a welder exposed to metal fumes. South Med J 92: 510-512.

18. Seidal K, Jörgensen N, Elinder CG, Sjögren B, Vahter M (1993) Fatal cadmiuminduced pneumonitis. Scand J Work Environ Health 19: 429-431.

19. Behbahani M, Tapeh NAG, Mahyari M, Pourali AR, Amin BG, et al. (2014) Monitoring of trace amounts of heavy metals in different food and water samples by flame atomic absorption spectrophotometer after preconcentration by amine-functionalized graphene nano sheet. Environ Monit Assess 186: 7245-7257.

20. Roychowdhury T, Tokunaga H, Ando M (2003) Survey of arsenic and other heavy metals in food composites and drinking water and estimation of dietary intake by the villagers from an arsenic-affected area of West Bengal, India. Sci Total Environ 308: 15-35.

21. Zheng J, Chen KH, Yan X, Chen SJ, Hu GC, et al. (2013) Heavy metals in food, house dust, and water from an e-waste recycling area in South China and the potential risk to human health. Ecotoxicol Environ Saf 96: 205-212.

22. Jarup L (2003) Hazards of heavy metal contamination. Br Med Bull. 68:167182.

23. Al-Saleh I, Shinwari N, Mashhour A, Mohamed Gel D, Rabah A (2011) Heavy metals (lead, cadmium and mercury) in maternal, cord blood and placenta of healthy women. Int J Hyg Environ Health 214: 79-101.

24. Pigatto PD, Minoia C, Ronchi A, Guzzi G (2013) Human placenta and markers of heavy metals exposure. Environ Health Perspect 121: A10.

25. Roels H, Hubermont G, Buchet JP, Lauwerys R (1978) Placental-transfer of lead, mercury, cadmium, and carbon-monoxide in women III. Factors influencing accumulation of heavy-metals in placenta and relationship between metal concentration in placenta and in maternal and cord blood. Environ Res. 16: $236-247$
26. Davit CJ, Hundley RJ, Bacic JD, Hanson EM (2011) A pilot study to improve venipuncture compliance in children and adolescents with autism spectrum disorders. J Dev Behav Pediatr 32: 521-525.

27. McDade TW, Williams S, Snodgrass JJ (2007) What a drop can do: dried blood spots as a minimally invasive method for integrating biomarkers into population-based research. Demography 44: 899-925.

28. Guthrie R, Susi A (1963) A simple phenylalanine method for detecting phenylketonuria in large populations of newborn infants. Pediatrics 32: 338343.

29. Olshan AF (2007) Meeting report: the use of newborn blood spots in environmental research: opportunities and challenges. Environ Health Perspect 115: $1767-1779$.

30. Funk WE, Waidyanatha S, Chaing SH, Rappaport SM (2008) Hemoglobin adducts of benzene oxide in neonatal and adult dried blood spots. Cancer Epidemiol Biomarkers Prev 17: 1896-1901.

31. Funk WE, McGee JK, Olshan AF, Ghio AJ (2013) Quantification of arsenic lead, mercury and cadmium in newborn dried blood spots. Biomarkers 18: 174 177 .

32. Pleil JD, Sobus JR, Stiegel MA, Hu D, Oliver KD, et al. (2014) Estimating common parameters of log normally distributed environmental and biomonitoring data: harmonizing disparate statistics from publications. J Toxicol Environ Health B, Crit Rev 17: 341-368.
This article was originally published in a special issue, Toxicology \& Environmental Safefy handled by Editor(s). Dr. Abdel-Tawab H. Mossa, National Research Centre, Egypt 